\title{
Co-trapping different species in ion traps using multiple radio frequencies
}

\author{
Dimitris Trypogeorgos ${ }^{*}$ and Christopher J. Foot \\ Clarendon Laboratory, Department of Physics, University of Oxford, \\ Parks Road, Oxford, OX1 3PU, UK
}

(Dated: July 17, 2016)

\begin{abstract}
We consider the stability of systems subjected to periodic parametric driving in the context of ions confined by oscillating electric fields. The most important aspects of these systems can be understood in terms of a pseudopotential approximation and resonances arising from parametric excitation. We introduce a novel linear Paul trap configuration operating with two radio frequencies to simultaneously confine two species with extremely different charge-to-mass ratios and investigate its key properties. The theoretical calculations have been verified by molecular dynamics simulations and normal modes analysis.
\end{abstract}

\section{INTRODUCTION}

Paul traps have been incredibly successful at confining charged particles, ranging from atomic ions to macroscopic charged objects [1]. The intrinsic sensitivity of the Paul trap mechanism on the charge-to-mass ratio of trapped particles can be exploited for mass spectrometry [2, 3]. While it is possible to simultaneously confine species with markedly different charge-to-mass ratios in a Paul trap, this results in the more weakly confined species being pushed away from the trap centre 4]. In this article, we describe a method of operating a Paul trap, with two radio-frequency $(\mathrm{RF})$ driving fields, in which two ions of extremely different charge-to-mass ratios experience harmonic pseudopotentials with similar spring constants [5]. In this way, both species can be tightly confined near the centre of the trap so that their interactions are enhanced. Although the additional driving frequency can induce more parametric resonances, it is possible to find parameter values where the system is unconditionally stable even in the absence of any applied damping. The addition of damping, from, e.g., laser or buffer gas cooling, acts to enlarge the stability region.

Gas-phase chemistry of ions at millikelvin temperatures involves the study of reaction rates and thermodynamic properties in the regime where quantum effects are important. Previous experiments and numerical simulations have used single frequency Paul traps and were limited to species with similar charge-to-mass ratios. Typically, the lighter species is a singly charged atomic ion, with a suitable laser cooling transition, co-trapped with a small molecule with mass of a few hundred amu [6]. Our scheme opens the way to working with much heavier charged particles such as biomolecules, nanodiamonds and graphene [7, 8]. Paul trapping of large biomolecular ions has also been discussed for DNA, but in an aqueous solution rather than the usual vacuum environment 9 .

The article is organised as follows. Section II summarises the well established theory of operation of the

\footnotetext{
* Currently at: Joint Quantum Institute, University of Maryland, College Park, Maryland, 20742, USA; E-mail: dtrypo@umd.edu
}

Paul trap in terms of the Mathieu equation and the pseudopotential approximation. The conditions for stable trapping are calculated using Floquet theory. We discuss parametric resonances and how they are affected by the addition of linear damping. In Section III we turn to the two-frequency Hill equation and show how the spring constants of two co-trapped species can be independently adjusted. In Section IV we present an example of co-trapping and sympathetically cooling a large charged particle with a handful of atomic ions. We use molecular dynamics and normal mode analysis to simulate the full dynamics of the system. Finally, we conclude in Section V] with a discussion of the experimental relevance of our results and future prospects.

\section{KINEMATICS OF THE PAUL TRAP}

The Paul trap, invented by Wolfgang Paul, confines ions with an oscillating electric quadrupole field [10, 11]. It has many diverse applications including frequency standards and quantum computing. The development of laser cooling techniques had an important impact on the use of ion traps, and even charged particles without suitable optical transitions can be cooled sympathetically by exchanging energy with atomic ions that are amenable to laser cooling techniques.

In the following we consider a linear Paul trap with alternating voltage $V(t)=V_{1} \cos \left(\Omega_{1} t\right)$ applied to four parallel rod-shaped electrodes and the end-caps held at a constant voltage $U_{0}$. The total electric field produced by such an arrangement is

$$
\mathbf{E}(\mathbf{x} ; t)=-V(t)\left(\frac{x \hat{\mathbf{x}}-y \hat{\mathbf{y}}}{R_{0}^{2}}\right)+\frac{U_{0}}{Z_{0}^{2}}(x \hat{\mathbf{x}}+y \hat{\mathbf{y}}-2 z \hat{\mathbf{z}})
$$

where $R_{0}, Z_{0}$ are the characteristic lengths along the radial and axial directions respectively.

\section{A. Mathieu equation}

The equation of motion for a single charged particle in the electric field of Eq. 1, including linear damping $\beta$, 


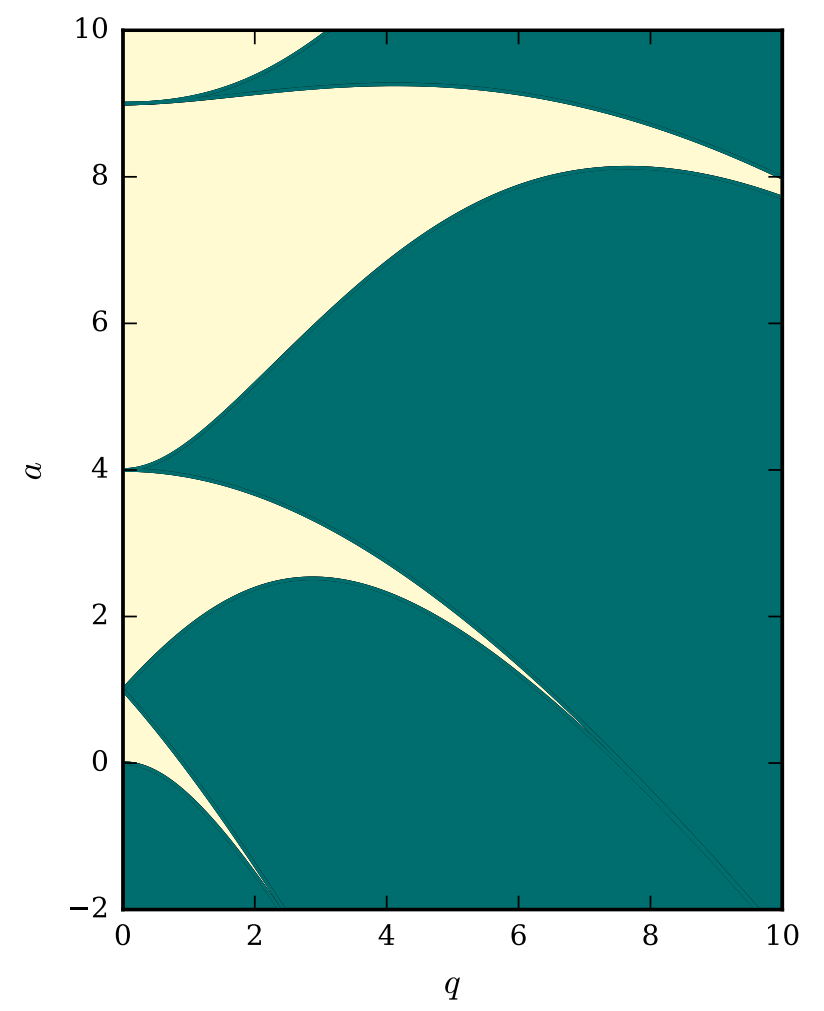

FIG. 1. The stability diagram of the Mathieu equation. The lightly coloured regions indicate where the system is stable. The diagram corresponds to a single transverse axis of a linear Paul trap. Full radial confinement is possible only where the stable regions for motion along the $x$ and $y$ axes overlap with each other. The usual operating regime for linear ion traps lies very close to the origin $a, q \ll 1$.

can be written in the form of a Mathieu equation [12, 13]

$$
\frac{d^{2} u_{i}}{d t_{1}^{2}}-\beta_{1} \frac{d u_{i}}{d t_{1}}+\left(a_{i}-2 q_{i} \cos \left(2 t_{1}\right)\right) u_{i}=0
$$

where $2 t_{1}=\Omega_{1} t$ is the effective time, $i$ indexes the three spatial coordinates and $\beta_{1}=2 \beta / \Omega_{1}$. The constants $a_{i}$ and $q_{i}$ depend on the ratio of charge $Q$ and mass $M$ of the trapped ion and the amplitudes of the static and oscillating voltage components:

$$
a_{x}=a_{y}=-\frac{1}{2} a_{z}=-\frac{4 Q U_{0}}{M Z_{0}^{2} \Omega_{1}^{2}}
$$

and similarly for $q$ :

$$
q_{x}=-q_{y}=\frac{2 Q V_{1}}{M R_{0}^{2} \Omega_{1}^{2}}, \quad q_{z}=0
$$

The non-singular solutions of Eq. 2 are the Mathieu characteristic functions 14 16. They are the elliptic equivalent of the trigonometric functions and were first discussed by Mathieu in 1868 [17, 18, in the context of finding the normal vibration modes of an elliptic membrane. An approximate solution to Eq. 2 can be found by separating the fast and slow oscillating components of the atomic motion, assuming the amplitude of the fast component is small. This is referred to as the pseudopotential approximation. The frequency of the secular motion of the ion is then related to the driving frequency by

$$
\omega_{i} \simeq \frac{\Omega_{1}}{2} \sqrt{a_{i}+\frac{1}{2} q_{i}^{2}}
$$

In the above we have assumed that the ion is unconditionally stable which is true for $a \ll 1$ and $q \leq 0.9$. This constitutes the first stability region of the Mathieu equation where ion traps normally operate. Fig. 1 shows the stability diagram for the one-dimensional Mathieu equation which is calculated using Floquet theory as described in Appendix A. Note that for the remainder of the article we only consider motion and stability along the $y$-axis and drop the $i$ subscript from the Mathieu coefficients.

\section{B. Damping and critical lines}

The condition for parametric resonance is $2 \omega=\Omega_{1} m$, where the integer $m$ is the order of the resonance. Parametric resonance causes the tongues of instability that emanate from the vertical axis in Figs. 1 and 2 (dark coloured regions). For $q=0$, the parametric resonances occur at $a=m^{2}$ as can be seen by inserting the resonance condition into Eq. 5. In the two-frequency trap parametric resonances arise from both driving frequencies, however if they are widely separated the behaviour can be interpreted in terms of the Mathieu equation albeit using values of $a$ and $q$ that are much higher than usually considered relevant to ion trapping. The stability diagram of the Mathieu equation shown in Fig. $2 \mathrm{a}$ includes the area shown in Fig. 1 but on a scale where the fine detail is not visible. For the large number of resonances shown in Fig. 2 there are obvious general features, i.e., the system is predominantly stable above the critical line $a=2 q$ and mostly unstable below [19].

The critical line is defined as the line that separates the stability diagram in regions of equal stable/unstable density [20, 21]. Since the Paul trap is normally operated at small values of $a$ and $q$, such a definition does not constitute a strict constraint from stable to unstable behaviour. A more useful criterion can be formulated by including damping.

The addition of damping enlarges the region where the system is stable as shown in Appendix $\mathrm{A}$. The parametric resonances now extend only to finite values of $q$, rather than $q=0$, as can be seen in Fig. 2 $\mathrm{b}$. The boundaries of the undamped resonance lie along $a=1 \pm q$ for $q \ll 1$ and $\beta_{1}=0$. These boundaries change with added damping and the threshold amplitude for exciting the parametric resonance becomes approximately equal to $\beta_{1}$. The 


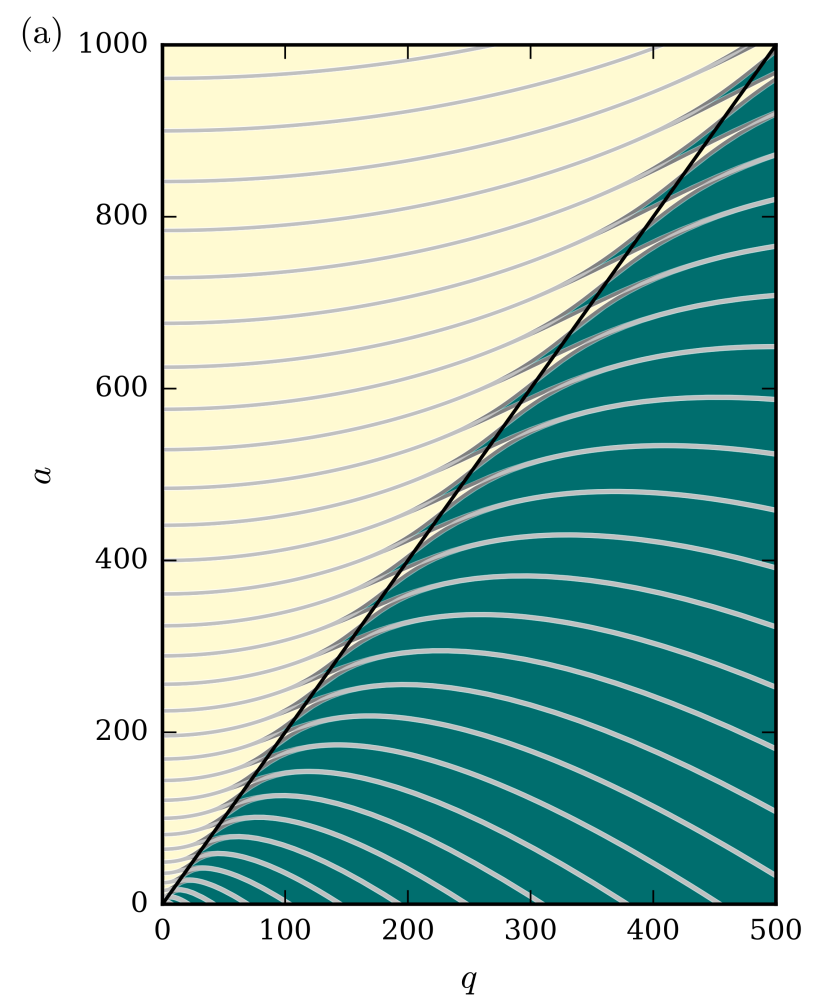

(b)

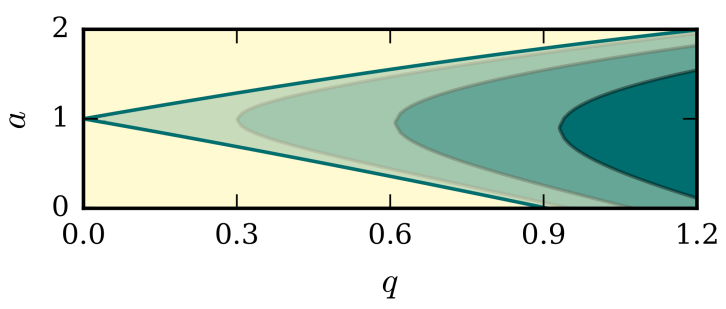

FIG. 2. (a) The stability diagram of the Mathieu equation for large $a$ and $q$. The resonances cutting through the stable region $a>2 q$ cause instability. They are drawn as lines of constant thickness for clarity, whereas in reality their width becomes vanishingly small. Similarly for the lines cutting through the predominantly unstable region. (b) A close-up of the first parametric resonance located at $a=1$. The boundaries of the undamped resonance lie along $a=1 \pm q$ for $q \ll 1$ and $\beta_{1}=0$. The addition of damping lifts the parametric resonances off of the $q=0$ axis, thus enlarging the stability region. The different shaded regions correspond, from left to right, to damping values $\beta_{1}=0.3,0.6,0.9$.

width of the instability tongues is the difference between the odd and even eigenvalues of the Mathieu equation which for $q \ll 1$ scales as $q^{m}\left(1+\mathcal{O}\left(q^{2}\right)\right)$ 22, 23]. The asymptotic behaviour of the width close to the $q=0$ line indicates that a small value of damping is sufficient to suppress the parametric resonance especially for higher order resonances. Damping guarantees that a resonance will not be excited unless the drive strength exceeds the threshold value

$$
\left.q\right|_{\mathrm{th} .}=C_{m}\left(\frac{\omega}{\Omega_{1}}\right)^{2}\left(\frac{\beta}{\omega}\right)^{1 / m}
$$

as has been shown in 24 26. Using the series expansion for the difference between odd and even eigenvalues of the Mathieu function the coefficient $C_{m}=$ $m^{-2}\left(2^{2 m-1}(m !)^{2}\right)^{1 / m}$.

\section{STABILITY OF THE TWO-FREQUENCY PAUL TRAP}

The equation of motion along the $y$-axis of a trapped ion subjected to an oscillating voltage of the form $V(t)=$ $V_{1} \cos \Omega_{1} t+V_{2} \cos \Omega_{2} t$ is

$$
\frac{d^{2} y}{d t_{1}^{2}}+\left(a_{1}-2 q_{1} \cos \left(2 t_{1}\right)-2 p_{1} \cos \left(2 n t_{1}\right)\right) y=0
$$

where time has been rescaled so that $\Omega_{1} t=2 t_{1}$ and the rest of the parameters are defined as for Eq. 2 with $q_{1} \propto V_{1}$ and $p_{1} \propto V_{2}$. The subscripts on the parameters indicate which driving frequency is being used to rescale Eq. 7. Here, the second driving frequency is a harmonic of the first $\Omega_{2}=n \Omega_{1}$, but fractional relations lead to similar results [27. To facilitate the presentation and interpretation of relevant results we consider only moderate values of $n$. The introduction of the driving frequency $\Omega_{1}$ leads to $n-1$ parametric resonances that slice through the previously stable regions as shown in Fig. 3 .

We identify two regimes of operation based on the value of $n$. When $n$ is small the parametric resonances are well defined and a stable configuration for the system can be found simply by avoiding them. Damping is less critical in this regime since stable points can be accessed by a judicious choice of system parameters. For higher values of $n$, the system enters a universal regime where the density of the instability tongues cutting through the stable region increases significantly, making it difficult to avoid them. However, the width of the resonances is exponentially small and they can be suppressed by modest amounts of damping as shown by Eq. 6. We then only need to consider the threshold excitation of the system to determine its stability. A similar picture arises if we take a slice of the stability diagram along the $a_{1}=0$ line on the $q_{1}-p_{1}$ plane (Fig. 4 ).

\section{A. Co-trapping two species}

Having established the criteria for stability we show how to simultaneously trap two species $M_{A}, M_{B}$ where $M_{B}>M_{A}$ with the two ion clouds overlapping. This can be achieved in a single-frequency trap only if the ions have the same $Q^{2} / M$ since the spring constant is $\kappa=M \omega^{2} \propto Q^{2} / M$. However, the charge-to-mass ratio generally decreases for heavy ions and putting them 

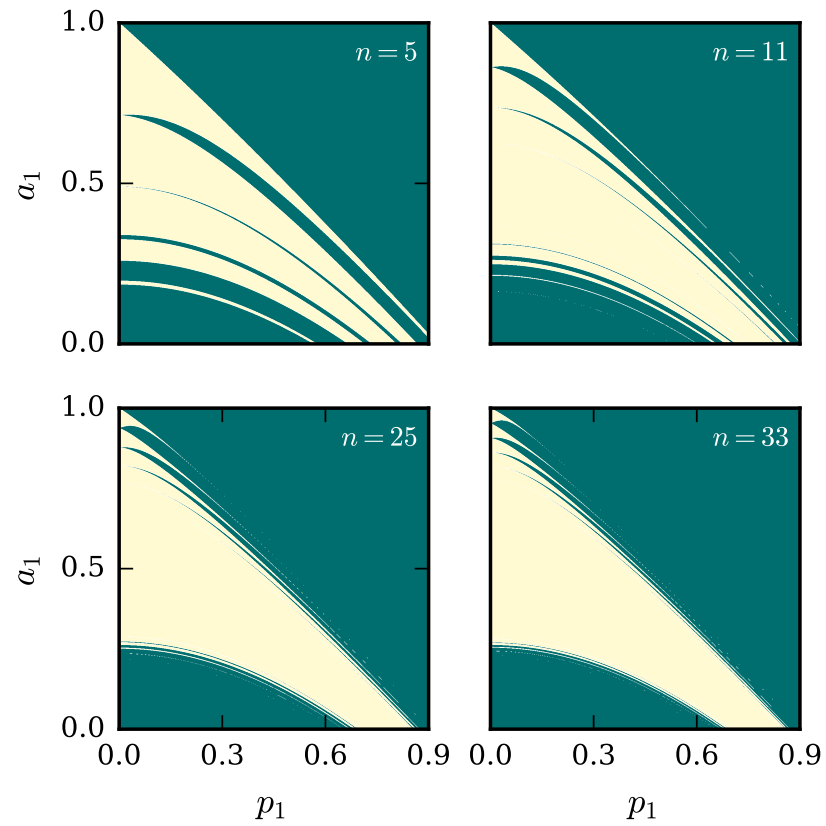

FIG. 3. The first region of stability of the two-frequency Hill equation on the $a_{1}-p_{1}$ plane for $n=5,11,25,33$ and $q_{1}=$ 0.04. Every panel contains $n-1$ parametric resonances, but for larger values of $n$ many of the resulting tongues are not visible at this resolution, since their width is exponentially small 28. Comparison with Fig. 1 shows that the second frequency causes instability within regions that were stable before.

into a higher charge state might not balance the spring constants because of the discretisation of charge. The required ratio of the dominant spring constants for the clouds to overlap is

$$
\frac{\kappa_{A}}{\kappa_{B}}=\left(\frac{V_{2} / \Omega_{2}}{V_{1} / \Omega_{1}}\right)^{2} \times \frac{Q_{A}^{2} / M_{A}}{Q_{B}^{2} / M_{B}} \simeq 1
$$

In previous works with single-frequency Paul traps the heavier ions were pushed to the outside of the atomic species cloud since $\kappa_{B} \ll \kappa_{A}$ [4, 6]. The two-frequency trap offers a significant improvement since the additional frequency dimension enables for the two species to have the same spring constants $\kappa_{A}=\kappa_{B}$, hence similar displacements from the centre of the trapping potential $\left|x_{A}\right| \approx\left|x_{B}\right|$ and larger overlap. By adjusting the values of $V_{i} / \omega_{i}$ in Eq. 8 we can achieve a precise balance of the spring constants for the two species so that both species congregate within a similar distance from the trap centre since by equipartition of energy, $\kappa\left\langle x^{2}\right\rangle \simeq k_{B} T$, for ion clouds at temperature $T$.

For $n \gg 1$ the system can be thought of as two individual nested Paul traps, since when the two frequencies are sufficiently different there exist conditions for which one of the oscillating terms can be considered as a minor perturbation acting on the system dominated by the other. The trap can be designed such that $\kappa_{B} \simeq \kappa_{B, 1}$
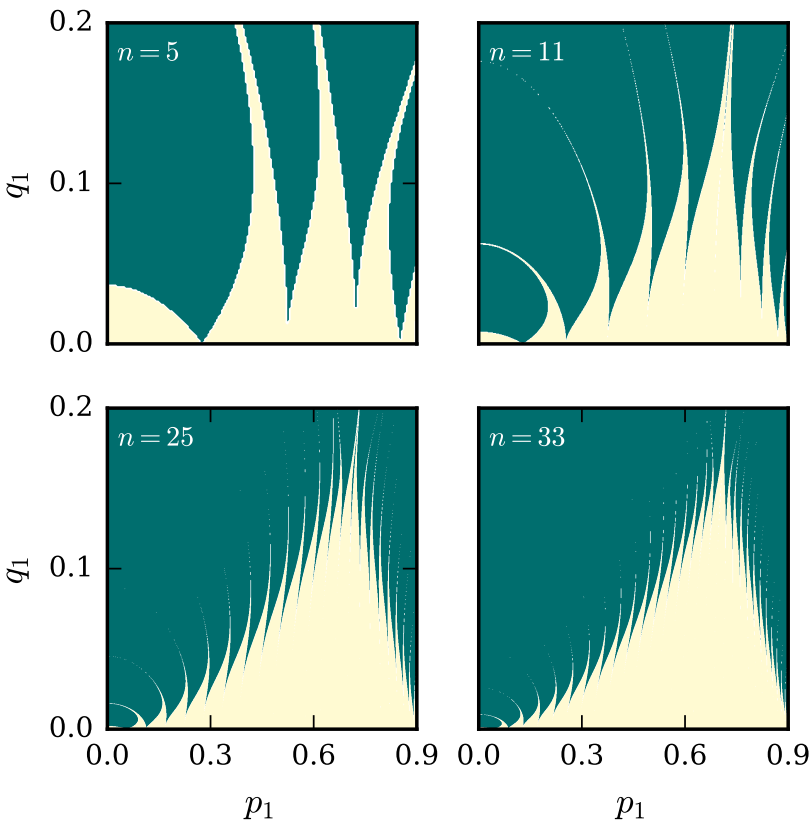

FIG. 4. The region of stability of the two-frequency Hill equation along $a=0$ on the $q_{1}-p_{1}$ plane for $n=5,11,25,33$. The system parameters can be chosen so that any parametric resonances are avoided for the top row. This is not the case for larger values of $n$ where the concept of a threshold line becomes useful.

and $\kappa_{A} \simeq \kappa_{A, 2}$ which indicates that the dominant contribution for the ion $M_{B}$ comes from the lower frequency $\Omega_{1}$, and the higher frequency $\Omega_{2} \gg \Omega_{1}$ mostly affects $M_{A}$. The field at $\Omega_{2}$ has negligible effect on $M_{B}$ since $\kappa_{B, 2} / \kappa_{B, 1} \ll 1$ and acts to increase trapping in any case. Thus for ions of species B, the two-frequency operation gives a pseudopotential very similar to a standard singlefrequency Paul trap. On the other hand, the fact that the variation of the trapping potential corresponding to $\Omega_{1}$ is slow enough to be adiabatic with respect to $\Omega_{2}$ implies that $\kappa_{A} \simeq \kappa_{A, 2}$. The quadrupole field at $\Omega_{1}$ induces additional parametric resonances.

Some constraints on the values of $V_{i}$ and $\Omega_{i}$ are imposed from the following considerations. The secular oscillation frequency of the light ions is $\omega_{A} \simeq q_{A} \Omega_{2} /(2 \sqrt{2})$. The frequency of this mechanical motion sets an upper limit for the lower radio frequency $\Omega_{1}$. The first parametric resonance occurs when the secular frequency of the system is half the slowest driving frequency. This implies a lower limit of the mass ratio at $M_{B} / M_{A} \geq n^{2}$. This limit can be established more precisely by numerical simulations in specific cases. The radio frequency $\Omega_{1}$ is chosen such that $\Omega_{1}<\omega_{A}$ in order to avoid the parametric excitation of species $M_{A}$ by the radio-frequency field that confines $M_{B}$. The resulting natural hierarchy of frequencies $\omega_{B}<\Omega_{1}<\omega_{A}<\Omega_{2}$ leads to two nested Paul traps capable of confining overlapping clouds of two species with very different charge-to-mass ratios. 


\section{EXAMPLE OF CO-TRAPPING AN ATOMIC ION WITH A BIOMOLECULE}

We demonstrate the feasibility of co-trapping atomic ${ }^{138} \mathrm{Ba}^{+}$ions with $M_{A}=140 \mathrm{amu}, Q_{A}=1$ and a heavy particle with $M_{B}=1.4 \times 10^{6} \mathrm{amu}, Q_{B}=33$, e.g. a nanoparticle or a macromolecule [29]. The choice of this extreme difference in charge-to-mass ratios fully illustrates the potential of this method. We choose $\Omega_{2}=2 \pi \times$ $10 \mathrm{MHz}$ as the main driving frequency. The maximum driving frequency ratio is set by $\Omega_{1}<\Omega_{2} \times\left(M_{A} / M_{B}\right)$. To achieve the same spring constant for both species we choose $n=100$ which makes $\Omega_{1}=2 \pi \times 100 \mathrm{kHz}$. The values of the applied voltages are chosen so that both species nominally experience a single-frequency Paul trap with $q \simeq 0.3$.

\section{A. Rescaling the equations of motion}

Appropriate rescaling of Eq. 7 is crucial for an intuitive understanding of the system. There are two ways of rescaling the equations of motion for each species, as shown in Table I. The intuitive physical picture that the heavy (light) ion is mainly affected by the slow (fast) frequency arises only when using the appropriate scaling for each atom.

TABLE I. Rescaling of the equations of motion for two species trapped in a two-frequency trap. Both species effectively experience a single frequency Paul trap with $q \simeq 0.3$ as indicated by the numbers in bold in the first and third row.

\begin{tabular}{cccc}
\hline \hline \multirow{2}{*}{$M_{B}, \Omega_{1}$} & $a_{B, 1}$ & $q_{B, 1}$ & $p_{B, 1}$ \\
& -0.0003 & $\mathbf{0 . 3 0 7}$ & 10.758 \\
\hline \multirow{2}{*}{$M_{B}, \Omega_{2}$} & $a_{B, 2}$ & $q_{B, 2}$ & $p_{B, 2}$ \\
& $-3.35 \times 10^{-8}$ & $2.952 \times 10^{-5}$ & 0.001 \\
\hline \multirow{2}{*}{$M_{A}, \Omega_{2}$} & $a_{A, 2}$ & $q_{A, 2}$ & $p_{A, 2}$ \\
& $-1.03 \times 10^{-5}$ & 0.009 & $\mathbf{0 . 3 1 8}$ \\
\hline \multirow{2}{*}{$M_{A}, \Omega_{1}$} & $a_{A, 1}$ & $q_{A, 1}$ & $p_{A, 1}$ \\
& -0.107 & 94.5 & 3307.4 \\
\hline \hline
\end{tabular}

Different physical pictures emerge depending on the choice of effective time $t_{1}$ or $t_{2}$ with the coefficients being rescaled with $n^{2}$, e.g., $q_{A, 2}=q_{A, 1} / n^{2}$. Species $M_{B}$ is mainly confined by the electric field at $\Omega_{1}$ with $q_{B, 1} \simeq 0.3$ as shown in the first row of Table I] Although $p_{B, 1}$ has a much larger value it does not significantly contribute to trapping. This becomes clear if we omit $\Omega_{1}$ and rescale time to $2 t_{2}=\Omega_{2} t$. The equation of motion for the species $M_{B}$ is then the Mathieu equation

$$
\frac{d^{2} y_{B}}{d t_{2}^{2}}+\left(a_{B, 2}-2 p_{B, 2} \cos \left(2 t_{2}\right)\right) y_{B}=0
$$

with $p_{B, 2}=0.001$, which provides negligible trapping (Table I, second row). The stability region for $M_{B}$ is thus only slightly perturbed by the presence of the high frequency term and the trapping comes predominantly from $\Omega_{1}$.

In a similar manner, $M_{A}$ is mainly trapped by $\Omega_{2}$ since $q_{A, 2} \ll p_{A, 2}$ (Table I third row). The effect of $\Omega_{1}$ on $M_{A}$ however, is more difficult to assess from Eq. 7] since $\Omega_{1}$ can excite parametric resonances. The system is stable as long as the amplitude of $q_{A, 2}$ does not exceed the threshold amplitude (see Sec. IVB).

The fourth row of Table@illustrates the connection between the large $a-q$ regime and the two-frequency driving. The large values of the coefficients are potentially misleading but, by treating the fast oscillating term as an effective harmonic pseudopotential term that arises from a static quadrupole, we can map Eq. 7 to the Mathieu equation

$$
\frac{d^{2} y_{A}}{d t_{1}^{2}}+\left(a_{\mathrm{eff}}-2 q_{A, 1} \cos \left(2 t_{1}\right)\right) y_{A}=0
$$

where $a_{\text {eff }}$ indicates the effective static potential term. The width of the induced parametric resonances decreases rapidly with increasing order number $m$ and thus the higher order resonances are readily suppressed by weak damping.

\section{B. Stability diagram}

Fig. 5 a shows a portion of the stability diagram of the system for $n=100$. The critical line can be calculated analytically to be $q_{1}=0.27 p_{1}^{2}$ for $0<p_{1}<0.7$. Shown in Fig. 5 a is the best fit line $q_{1}=-0.002+0.29 p_{1}^{2}$. Numerical calculations for $0.7<p_{1}<0.9$ show that the critical line has a maximum at $p_{1}=0.7$ and can be approximated by $q_{1}=0.57\left(0.9-p_{1}\right)$. The stability region extends up to $p_{1}=0.9$ as in the Mathieu equation. Parametric excitation of $M_{A}$ by $\Omega_{1}$ produces tongues of instability which are too fine for the numeric calculations to capture. Since the light ions experience a pseudopotential with secular frequency $\omega \approx p_{A, 2} \Omega_{2} /(2 \sqrt{2})$ we can use Eq. 6 to put an upper limit to the amplitude of the $\Omega_{1}$ term

$$
\left.q_{A, 2}\right|_{\mathrm{th} .}=\frac{2 p_{A, 2}^{2}}{e^{2}}\left(\frac{\pi \beta}{\Omega_{1}}\right)^{1 / m}
$$

where the order of the resonance is $m=p_{A, 2} \Omega_{2} /\left(\sqrt{2} \Omega_{1}\right)$ and using Stirling's approximation for $m$ !. For $n=$ $\Omega_{2} / \Omega_{1}=100$ and $p_{A, 2}=0.32$ the parametric resonance happens at $m=23$. For this high order resonance even light damping $\beta / \Omega_{1}=10^{-6}$ leads to a threshold value $q_{A, 2}=0.016$ which is comfortably above our chosen operating value $q_{A, 2}=0.01$ (see Fig. 5a). For even larger driving frequency ratios, the lower frequency can be considered as DC compared to the secular motion of the lighter species leading to the stability criterion $q_{A, 2}<0.5 p_{A, 2}^{2}$. 

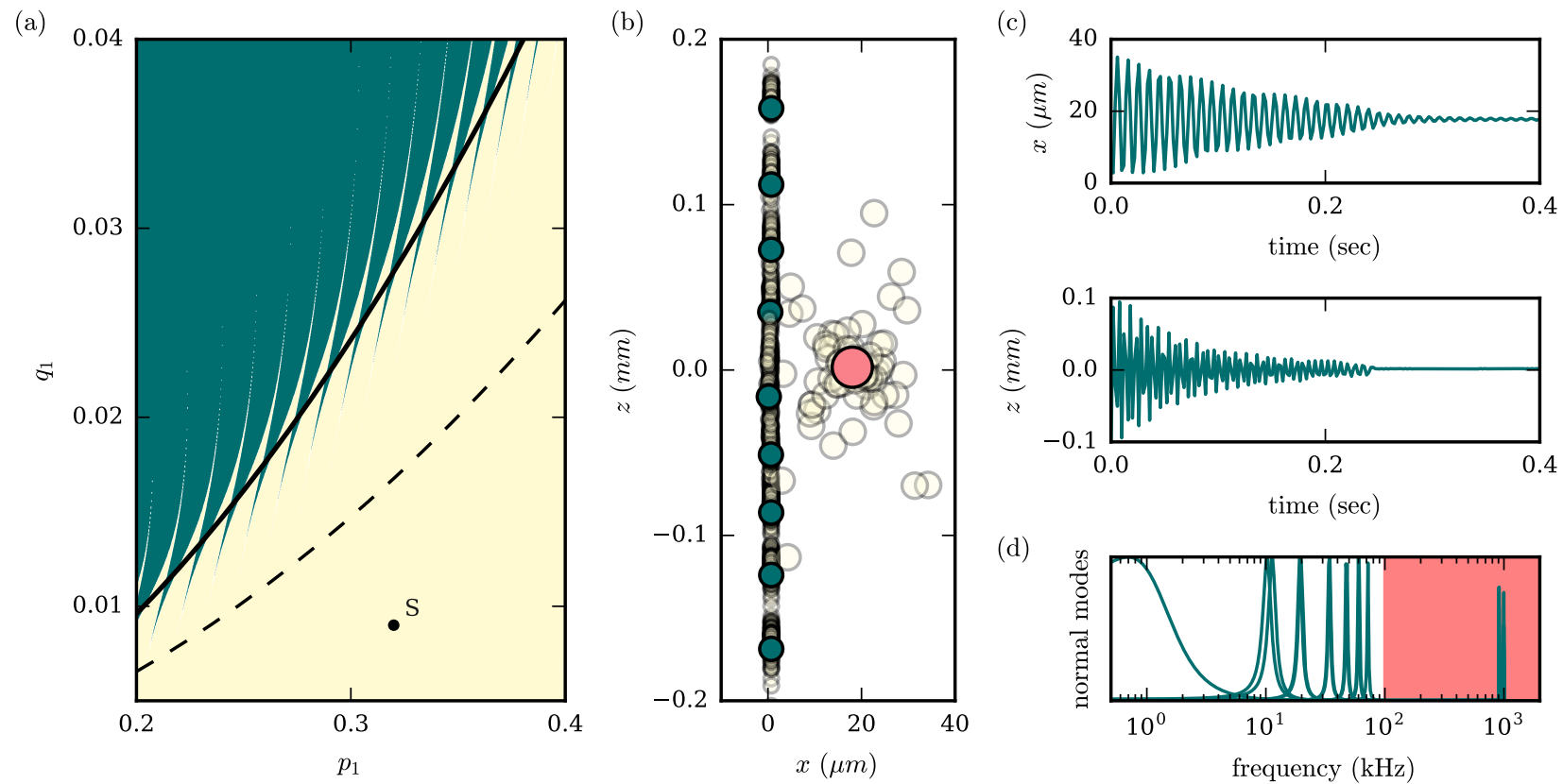

(d)

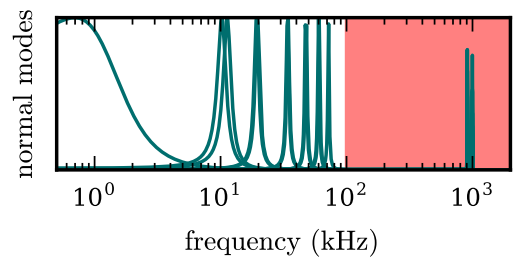

FIG. 5. (a) Part of the stability region for the ${ }^{138} \mathrm{Ba}^{+}$ions. The point $S=(0.318,0.009)$ corresponds to the parameters used to give stable confinement. The tongues of instability extend from the cusps down to the horizontal axis, however, they are not visible due to the finite resolution of the plot even in the absence of any applied damping. The critical line (solid black) is the best fit line $q_{1}=-0.002+0.29 p_{1}^{2}$ for this region. The dashed line corresponds to the threshold drive needed to excite a resonance as calculated by Eq. 11. (b) An example configuration of light ions arranged in a linear chain with the heavy ion (red) is displaced from the axis due to $E_{\perp}$. (c) Characteristic trajectory of the heavy ion. Both axial and radial motion are damped at similar timescales of a few hundred ms. (d) The spectral density of the normal modes of the system. The mid-frequency normal modes are not allowed to cross into the region highlighted in red to avoid parametric excitation by $\Omega_{1}$.

\section{Numerical results}

We have carried out extensive numerical simulations using the open-source software package LAMMPS 30] to verify that the ions are confined in all three dimensions by a quadrupole field oscillating with two frequencies. Using the single-ion case as a guide for the stability region of the system we simulated the full dynamics of the equations of motion

$$
M_{j} \ddot{\mathbf{x}}_{j}=\left(E\left(\mathbf{x}_{j} ; t\right)+E_{\perp}\right) Q_{j}+\sum_{\substack{i, j=1 \\ i \neq j}}^{N} \frac{1}{8 \pi \epsilon_{0}} \frac{Q_{i} Q_{j}}{\left(\mathbf{x}_{j}-\mathbf{x}_{i}\right)^{2}}
$$

for ion $j$, where $E\left(\mathbf{x}_{j} ; t\right)$ is the electric field from Eq. 1 with $V(t)=V_{1} \cos \Omega_{1} t+V_{2} \cos \Omega_{2} t, E_{\perp}$ is an optional DC transverse electric field and $\epsilon_{0}$ is the permittivity of vacuum. The full-range Coulomb interaction is included.

For our choice of parameters, the radial and axial oscillation frequencies for $M_{B}$ are $11.4 \mathrm{kHz}$ and $0.7 \mathrm{kHz}$ respectively. For $M_{A}$, the radial frequency is $\omega_{A}=$ $2 \pi \times 1 \mathrm{MHz}$ and $\omega_{A, z}=\omega_{B}$ so as to promote resonant energy transfer between the two species. This frequency matching is easily achieved when the number of ions and hence the number of normal modes of the system becomes larger. We have explored the behaviour of the system for different values of $E_{\perp}$ and different numbers of ions and have always observed energy being exchanged between the two species.

The radial electric field $E_{\perp}$ breaks the symmetry of the system and couples the radial and axial motion of the ions which leads to more efficient energy exchange between the large ion and the linear chain of smaller ones (Fig. 5p). This needs to be balanced against RFinduced heating due to the ions being displaced from the RF-null nodal line and thus experiencing larger micromotion. However, the system is tolerant against heating due to the much larger Coulomb interactions. The crystallisation transition temperature of the Coulomb crystal is much higher than typical atomic systems, as can be estimated using the Lindemann criterion by equating the average amplitude of the thermal vibrations of the ions to their mean separation. This leads to a transition temperatures in the regime of a few $\mathrm{mK}$, that is, larger by a factor of $Q_{B}=33$ over single species atomic crystals.

We have simulated various configurations involving up to 10 Doppler cooled ${ }^{138} \mathrm{Ba}^{+}$ions and a single ion of species B. We observed that the damping rate depends strongly on the size and degree of asymmetry of the Coulomb crystal. The axial asymmetry of the crystal was varied by changing the initial position of the heavy ion. More asymmetric crystals tend to cool down faster 
with cooling times in the range of $100 \mathrm{~ms}$ to $1 \mathrm{~s}$, inversely proportional to the number of ions. The field $E_{\perp}$ can be used to improve the coupling of the translational motion of $M_{B}$ to the normal modes of the light ion chain. Due to the very different secular frequencies of the two species, the out-of-plane motion of $M_{B}$ is cooled less efficiently and limits the overall cooling rate. The optimal value for $E_{\perp}$ can be calculated within the pseudopotential approximation to be in the intermediate regime where a linear chain of $N$ ions transitions to an effective $N-1$ chain with the heavy ion displaced off axis. However, RF micromotion heating limits the maximum value of $E_{\perp}$ to much lower values.

Fig. 5r shows that $1 / e$ cooling times of about $200 \mathrm{~ms}$ can be achieved for a crystal of 10 ions. Additional $M_{A}$ ions position themselves approximately along the z-axis and start forming a chain since $\omega_{A} \gg \omega_{A, z}$. The axial modes of such a linear Coulomb crystal have higher frequency than the centre-of-mass mode whereas the opposite is true for higher-order radial modes (see Appendix B . Adding more ions tends to close the gap in the frequency spectrum of normal modes close to $\Omega_{1}$ (see Fig. $5 \mathrm{~d}$ ). This limits the number of ions of species A that can be accommodated in the same potential well, as an overlap of normal modes with $\Omega_{1}$ could lead to resonant heating. Larger systems can still be stable by operating at smaller values of $n$ or increasing the damping for all species, e.g. with buffer gas cooling.

\section{CONCLUSIONS}

We presented calculations on the two-frequency operation of an ion trap carried out within the mathematical framework of Floquet theory. Using this as a guide for the stability of the system, we demonstrated the feasibility of confining different species of ions with the same effective spring constant using molecular dynamics simulations. We specifically chose an extremely different charge-to-mass ratio to demonstrate the usefulness of the two-frequency operation of the trap. Pushing the chargeto-mass ratio even further apart is possible by applying more than two frequencies. However sympathetic cooling is likely to be more difficult to achieve, as the frequencies of the axial and radial normal modes become more separated. Our method also works for lower charge-to-mass ratios but it will be more susceptible to normal mode spectral crowding.

Our numerical simulations show how a typical megadalton charged particle can be sympathetically cooled by atomic ${ }^{138} \mathrm{Ba}^{+}$ions. The presence of the nonfluorescing, dark ion can be deduced from its large effect on the positions of the observable atomic ions. Atomic ions scatter laser light so that individual fluorescing ions can be observed, thus permitting indirect detection of dark ions as holes in the cloud. Pre-cooling can be achieved by means of the conventional single-frequency operation of a linear Paul trap [6]. However, with a sin- gle frequency the trapping of species B is very weak so these ions might be destabilised by either repulsion from the atomic ions or the radial component of the axial DC field and a second driving frequency is required for stable trapping.

Many interesting possibilities arise from being able to extend laser techniques for sympathetic cooling of both the translational and rotational degrees of freedom of large objects that are not amenable to laser cooling like viruses, molecular motors and dust particles [29, 31, 32. Cooling mesoscopic objects like nanodiamonds to their quantum ground state and adapting the sophisticated techniques developed for quantum information processing with trapped ions will allow for investigating entanglement and decoherence dynamics. Reversing the role of the ions, antimatter can be trapped and efficient cooled as has been shown previously only in Penning traps 33 . Our work inspired a re-examination of these ideas for trapping antihydrogen [27.

\section{ACKNOWLEDGMENTS}

The authors would like to acknowledge the use of the Oxford Supercomputing Centre in carrying out this work. We acknowledge funding from the Bodossaki Foundation and St. Peter's College (DT) and partial support from the EPSRC. The research leading to these results is supported by EU through the Collaborative Project QuProCS (Grant Agreement No. 641277). We thank Elliot Bentine for useful discussions, reading the manuscript and work on LAMMPS.

\section{Appendix A: Differential equations with periodic coefficients}

Ordinary differential equations (ODEs) with periodic coefficients that contain an arbitrary number of frequencies in their Fourier spectrum are ubiquitous in physics. They describe the temporal behaviour of driven systems or the spatial character of the wavefunction in Hamiltonians of crystalline structures, e.g. driven atomic systems, Bloch wavefunctions and mechanical vibrations [34 36]. Linear second order homogeneous ODEs with periodic coefficients have the form

$$
\frac{d^{2} u}{d t^{2}}+B(t) \frac{d u}{d t}+S(t) u=0
$$

with $B(t+T)=B(t)$ and $S(t+T)=S(t)$ where $T$ is the period of the system. Since any function can be expressed in terms of even and odd functions without loss of generality we choose $S(-t)=S(t)$ and rewrite the $S(t)$ term in Eq. A1 equation through its cosine Fourier representation to give

$$
\frac{d^{2} u}{d t^{2}}+\left(c_{0}+2 \sum_{n=1}^{\infty} c_{n} \cos (2 n t)\right) u=0
$$


where $B(t)=0$. Calculating the characteristic exponents of Eq. A2 allows us to map out the stability diagram for the phase space spanned by the parameters $c_{n}$. The Mathieu equation is a particular case of Eq. A2 with only the constant and first oscillatory term being non-zero.

\section{Floquet theory}

The Floquet formalism can be used to solve ODEs with periodic coefficients [28, 37. We recast Eq. A1 as a system of first order equations

$$
\frac{d}{d t} \mathbf{X}(t)=\left(\begin{array}{cc}
0 & 1 \\
-S(t) & -B(t)
\end{array}\right) \mathbf{X}(t)=0
$$

where $\mathbf{X}(t)=(x(t), \dot{x}(t))^{T}$. The stability of the system can be analysed by looking at the value of the propagation matrix $M=\left(\mathbf{X}_{1}(T), \mathbf{X}_{2}(T)\right)$ after time equal to a period $T$ has transpired. At $t=0$ the propagation matrix equals the identity $M=I$ so that its Wronskian is zero and $\mathbf{X}_{1}, \mathbf{X}_{2}$ are fundamental solutions of the system. The characteristic equation $\operatorname{det}\{M-\lambda I\}$ of the system has solutions $\lambda_{1,2}$ given by its characteristic polynomial

$$
\lambda_{1,2}=\frac{1}{2}\left(\operatorname{tr}\{M\} \pm\left(\operatorname{tr}\{M\}^{2}-4 \operatorname{det}\{M\}\right)^{1 / 2}\right)
$$

and the determinant of $\mathrm{M}$ is

$$
\operatorname{det}\{M\}=\lambda_{1} \lambda_{2}=\exp \left(\int_{0}^{T}-B(\tau) \mathrm{d} \tau\right)
$$

For the case where $B(t)=0$ the determinant becomes equal to 1 . This is a general property of symplectic matrices that is directly related to the Liouville theorem and expresses the preservation of phase space for a dynamic system. For the system to be stable its eigenvalues must be inside the unit circle in the complex plane $\max \left\{\left|\lambda_{1}\right|,\left|\lambda_{2}\right|\right\} \leq 1$, or equivalently $|\operatorname{tr}\{M\}| \leq 2$. The trace-determinant plane fully characterises the stability of the system. When $|\operatorname{tr}\{M\}|>2$ there are two real eigenvalues. Their product is $\lambda_{1} \lambda_{2}=1$ hence either $\lambda_{1}>1$ or $\lambda_{2}>1$, giving an unbounded, exponentially diverging solution. On the other hand, when $|\operatorname{tr}\{M\}| \leq 2$ there are two complex eigenvalues, with $\left|\lambda_{1}\right|=\left|\lambda_{2}\right|=1$ and $\lambda_{1}=\lambda_{2}^{*}$. They can be written in the form $\lambda=e^{ \pm \mathrm{i} \theta}$ so that $\operatorname{tr}\{M\}=2 \cos \theta$. These correspond to stable, bounded solutions. In effect, knowledge of the sign of the discriminant $\Delta=\operatorname{tr}\{M\}^{2}-4$ is enough to determine the behaviour of the system. The stability points of the undamped Mathieu equation lie along the $\operatorname{det}\{M\}=1$ line and, if stable, are bounded by the parabola $\operatorname{tr}\{M\}^{2}=4 \operatorname{det}\{M\}$. Along the transition curves at the boundary of the above regions $\operatorname{tr}\{M\}= \pm 2$ and the discriminant is zero. The characteristic equation has a double root and degenerate eigenvalues $\lambda_{1}=\lambda_{2}= \pm 1$ corresponding to the system oscillating with period $T$ or $2 T$ respectively.

\section{Damping}

For constant damping $B(t)=\beta>0$, the stability condition becomes $\left|\operatorname{tr}\left\{M^{\prime}\right\}\right| \leq 1+e^{-\beta T}$ where we have written the propagation matrix as $M^{\prime}$ to differentiate it from the undamped system. At first sight this equation seems counterintuitive since it appears to reduce the limit on the magnitude of $\operatorname{tr}\left\{M^{\prime}\right\}$. However, the stability region is actually enlarged since $\operatorname{det}\left\{M^{\prime}\right\}=e^{-\beta T}$ rather than 1 as in the undamped case. The product of the eigenvalues in this case is bounded by a circle of radius $e^{-\beta T}$ in the complex plane. The point where one of the eigenvalues becomes greater than 1 is $e^{\beta T}+1$ as can be seen by direct substitution to Eq. A4.

To compare the two cases we can assume that $\lambda$ is the eigenvalue of a matrix of the same form as for the undamped equations $M$, but with $\omega^{2} \rightarrow \omega^{2}-(\beta)^{2}$. This leads to the stability condition $\max \left\{\left|\lambda_{1}\right|,\left|\lambda_{2}\right|\right\} \leq e^{\beta T}$. The damping term factors out when taking the trace of the propagation matrix, so that $M^{\prime}=e^{-\beta T} M$. Although the damped system can be investigated directly its behaviour is determined straightforwardly from the corresponding undamped system [38, 39].

\section{Appendix B: Normal modes of motion of damped, stiff systems}

A system is defined as stiff when at least one of the parameters describing it can take extremely different values that lead to rapid variations in the solution. This is the case when calculating the normal modes of motion of a two-species ion chain where the mass of one species is much larger than the other. Moreover, the addition of damping leads to a matrix equation that is not an eigenvalue equation. Here we show how the matrix equations can be recast to an eigenvalue equation which can be solved using efficient numerical methods.

First we define the pseudopotential for a chain of $N$ ions in a linear Paul trap including the Coulomb interaction

$$
\begin{aligned}
V(\mathbf{x}) & =\sum_{j=1}^{N} \frac{1}{2} M_{j} \omega_{r, j}^{2}\left(x_{j}^{2}+y_{j}^{2}\right)+Q_{j} E_{\perp} x_{j}+ \\
& +\sum_{j=1}^{N} \frac{1}{2} M_{j} \omega_{z, j}^{2} z_{j}^{2}+\sum_{\substack{i, j=1 \\
i \neq j}}^{N} \frac{1}{8 \pi \epsilon_{0}} \frac{Q_{i} Q_{j}}{\left|\mathbf{x}_{j}-\mathbf{x}_{i}\right|}
\end{aligned}
$$

with the trap frequencies defined as in Eq. 5 We can find the equilibrium positions by minimising the above potential [40. The field $E_{\perp}$ not only displaces the atoms along the radial plane but also alters the coupling between the modes. The system is effectively described as a system of coupled oscillators with the restoring forces for each particle arising through the competition between the trapping potential and the Coulomb repulsion of the ions. The equations of motion can be written in matrix 
form and assuming oscillatory solutions we can calculate the normal modes from the following determinant

$$
\operatorname{det}\left\{M \omega^{2}+G \omega+K\right\}=0
$$

where $M$ is the mass matrix, $G$ is the damping matrix and $K$ is the Hessian of the system. We refer to $G$ as classical damping matrix if $P^{T} G P$ is diagonal, where $P$ is matrix of the eigenvectors of the Hessian. The equations of motion are then uncoupled and the damping matrix can be factored into the Hessian and mass matrices.

To treat more generalised (non-classical) damping we follow [41, whereby the problem of solving the determinant equation is transformed into an eigenvalue problem in the $2 N$ space. The normal modes correspond to the eigenvalues of the determinant of the system given by the eigenvalue equation

$$
\left(I \omega^{-1}+U\right) \mathbf{Z}=0
$$

where $\mathbf{Z}=\exp (-\omega t) \mathbf{X}, \mathbf{X}=(\{\dot{\mathbf{x}}\},\{\mathbf{x}\})^{T}$ with dimensions $2 N \times 1$ and $I$ is the identity matrix. The block matrix $U$ takes the form

$$
U=\left(\begin{array}{cc}
0 & I \\
-K^{-1} M & -K^{-1} G
\end{array}\right)
$$

The eigenvalues of the system correspond to velocityposition pairs. The mode frequencies and characteristic damping times correspond to the inverse of the imaginary and real parts of $\mathbf{x}$ respectively.
[1] W. M. Itano, J. C. Bergquist, J. J. Bollinger, and D. J. Wineland, Physica Scripta 1995, 106 (1995).

[2] R. E. March and J. F. J. Todd, Quadrupole Ion Trap Mass Spectrometry, Volume 165, Second Edition|(WileyVCH, 2005).

[3] P. K. Ghosh, Ion Traps (Oxford University Press, Oxford : New York, 1996).

[4] D. Offenberg, C. B. Zhang, C. Wellers, B. Roth, and S. Schiller, Physical Review A 78, 061401 (2008).

[5] A preliminary discussion on two-frequency traps appears in H. Dehmelt, Physica Scripta 1995, 423 (1995).

[6] A. Ostendorf, C. B. Zhang, M. A. Wilson, D. Offenberg, B. Roth, and S. Schiller, Physical Review Letters 97, 243005 (2006)

[7] S. Willitsch, M. T. Bell, A. D. Gingell, and T. P. Softley, Physical Chemistry Chemical Physics 10, 7200 (2008)

[8] B. E. Kane, Physical Review B 82, 115441 (2010),

[9] X. Zhao and P. S. Krstic, Nanotechnology 19, 195702 (2008).

[10] Wolfgang Pauli humorously referred to him as his "imaginary part", G. E. Brown and C.-H. Lee, Hans Bethe and His Physics (World Scientific, 2006).

[11] W. Paul, Reviews of Modern Physics 62, 531 (1990).

[12] W. Paul, Angewandte Chemie International Edition in English 29, 739 (1990)

[13] D. Wineland, C. Monroe, W. Itano, D. Leibfried, B. King, and D. Meekhof, Journal of Research of the National Institute of Standards and Technology 103, 259 (1998)

[14] G. B. Arfken and H.-J. Weber, Mathematical methods for physicists (Elsevier, Boston, 2005).

[15] L. Ruby, American Journal of Physics 64, 39 (1996).

[16] N. W. McLachlan, Theory and Application of Mathieu Functions (Dover).

[17] E. Mathieu, Journal de Mathématiques Pures et Appliquées , 137 (1868).

[18] E. T. Whittaker, A Course of Modern Analysis, 4th ed. (Book Jungle, 2009).

[19] M. I. Weinstein and J. B. Keller, SIAM Journal on Applied Mathematics 47, 941 (1987) ArticleType: research-article / Full publication date: Oct., 1987 / Copyright 1987 Society for Industrial and Applied Math- ematics.

[20] H. Broer and M. Levi, Archive for Rational Mechanics and Analysis 131, 225 (1995).

[21] H. Broer, M. Levi, and C. Simo, Nonlinearity 26, 565 (2013)

[22] DLMF, "NIST Digital Library of Mathematical Functions," http://dlmf.nist.gov/, Release 1.0.10 of 2015-0807, online companion to 23].

[23] F. W. J. Olver, D. W. Lozier, R. F. Boisvert, and C. W. Clark, eds., NIST Handbook of Mathematical Functions (Cambridge University Press, New York, NY, 2010) print companion to 22 .

[24] X. Zhao, V. L. Ryjkov, and H. A. Schuessler, Physical Review A 66, 063414 (2002).

[25] M. A. N. Razvi, X. Z. Chu, R. Alheit, G. Werth, and R. Blümel, Physical Review A 58, R34 (1998)

[26] C. Foot, E. Bentine, D. Trypogeorgos, A. Gardner, and M. Keller, in preparation (2016).

[27] N. Leefer, K. Krimmel, W. Bertsche, D. Budker, J. Fajans, R. Folman, H. Haeffner, and F. SchmidtKaler, arXiv:1603.09444 [hep-ex, physics:physics] (2016), arXiv: 1603.09444.

[28] N. V. Konenkov, M. Sudakov, and D. J. Douglas, Journal of the American Society for Mass Spectrometry 13, 597 (2002)

[29] J. L. P. Benesch, B. T. Ruotolo, D. A. Simmons, and C. V. Robinson, Chemical reviews 107, 3544 (2007)

[30] S. Plimpton, Journal of Computational Physics 117, 1 (1995) (py)LIon: a wrapper for LAMMPS was used for the numerical calculations and is described in E. Bentine and D. Trypogeorgos, in preparation (2016).

[31] S. Kahra, G. Leschhorn, M. Kowalewski, A. Schiffrin, E. Bothschafter, W. Fues, R. de Vivie-Riedle, R. Ernstorfer, F. Krausz, R. Kienberger, and T. Schaetz, Nature Physics 8, 238 (2012)

[32] P. F. Staanum, K. Højbjerre, P. S. Skyt, A. K. Hansen, and M. Drewsen, Nature Physics 6, 271 (2010)

[33] B. M. Jelenkovic, A. S. Newbury, J. J. Bollinger, W. M. Itano, and T. B. Mitchell, Physical Review A 67, 063406 (2003)

[34] C. J. Foot, Atomic Physics (OUP Oxford, 2004).

[35] N. W. Ashcroft and N. D. Mermin, Solid state physics 
(Harcourt College Publishers, New York; London, 1976).

[36] J. H. Shirley, Physical Review 138, B979 (1965)

[37] D. Jordan and P. Smith, Nonlinear Ordinary Differential Equations: An Introduction for Scientists and Engineers, 4th ed. (OUP Oxford, 2007).

[38] M. Nasse and C. Foot, European Journal of Physics 22,
$563(2001)$

[39] T. Hasegawa and K. Uehara, Applied Physics B Laser and Optics 61, 159 (1995).

[40] D. F. V. James, Applied Physics B 66, 181 (1998).

[41] M. E. J. O'Kelly, Normal modes in damped systems, engd, California Institute of Technology (1961). 